\title{
Medical Image Diagnosis of Lung Cancer by Deep Feedback GMDH-Type Neural Network
}

\author{
Tadashi Kondo ${ }^{\dagger}$ \\ Graduate School of Health Sciences, Tokushima University \\ 3-18-15 Kuramoto-cho Tokushima 770-8509 Japan \\ Email:"kondomedsci@gmail.com \\ Junji Ueno \\ Graduate School of Health Sciences, Tokushima University \\ 3-18-15 Kuramoto-cho Tokushima 770-8509 Japan \\ Shoichiro Takao \\ Graduate School of Health Sciences, Tokushima University \\ 3-18-15 Kuramoto-cho Tokushima 770-8509 Japan
}

\begin{abstract}
The deep feedback Group Method of Data Handling (GMDH)-type neural network is applied to the medical image diagnosis of lung cancer. The deep feedback GMDH-type neural network can identified very complex nonlinear systems using heuristic self-organization method which is a type of evolutionary computation. The deep neural network architectures are organized so as to minimize the prediction error criterion defined as Akaike's Information Criterion (AIC) or Prediction Sum of Squares (PSS). In this algorithm, the principal component-regression analysis is used for the learning calculation of the neural network. It is shown that the deep feedback GMDH-type neural network algorithm is useful for the medical image diagnosis of lung cancer because deep neural network architectures are automatically organized using only input and output data.
\end{abstract}

Keywords: Deep neural networks, GMDH, Medical image diagnosis, Evolutionary computation, lung cancer

\section{Introduction}

The deep GMDH-type neural network algorithms were proposed in our early works $^{1 \sim 4}$ and can automatically organize the neural network architectures using heuristic self-organization method ${ }^{5,6}$ which is a type of the evolutionary computation. In this study, deep feedback GMDH-type neural network algorithm ${ }^{1}$ is applied to the medical image diagnosis of lung cancer. The learning calculations of the weights is the principal component-regression analysis. In previous our work ${ }^{3}$, we applied the logistic GMDH-type neural network to the medical image diagnosis of lung cancer. First, the lung regions were recognized by the logistic GMDHtype neural network and these lung regions were extracted and then the lung cancer regions were extracted using the image post processing of the extracted lung regions. In another previous our work ${ }^{4}$, we applied the hybrid feedback GMDH-type neural network to medical image diagnosis of liver cancer. The liver cancer regions were recognized and extracted using the same image processing using the hybrid feedback GMDH-type neural network.

In this paper, we apply the deep feedback GMDHtype neural network and the lung cancer regions are recognized directly using the deep feedback GMDHtype neural network and the lung cancer regions are extracted accurately. The recognition results shows that the deep feedback GMDH-type neural network can recognize the lung cancer regions directly and extract accurately. 


\section{Deep feedback GMDH-type neural network ${ }^{1}$}

The architecture of the deep feedback GMDH-type neural network used in this paper has a feedback loop as shown in Fig.1.

\subsection{First loop calculation}

First, all data are set to the training data. Then the architecture of the input layer is organized.

\subsubsection{Input layer}

$$
u_{j}=x_{j} \quad(j=1,2, \ldots, p)
$$

where $x_{j}(j=1,2, \ldots, p)$ are the input variables of the system, and $p$ is the number of input variables.

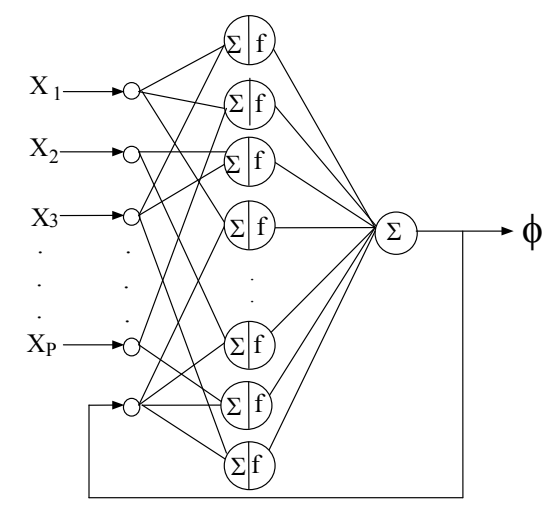

Fig.1 Architecture of the deep feedback GMDH-type neural network

\subsubsection{Hidden layer}

All combinations of the $r$ input variables are generated. For each combination, three types of neuron architectures which are the sigmoid function neuron, the redial basis function (RBF) neuron and the polynomial neuron, are generated and $L$ neurons which minimize $\mathrm{AIC}^{7}$ and $\mathrm{PSS}^{8}$ value are selected for each type of neuron architectures.

\section{(1) Sigmoid function neural network}

\section{1) The first type neuron}

$\Sigma:$ (Nonlinear function)

$$
z_{k}=w_{l} u_{i}+w_{2} u_{j}+w_{3} u_{i} u_{j}+w_{4} u_{i}^{2}+w_{5} u_{j}^{2}-w_{0} \theta_{l}
$$

$f:$ (Nonlinear function)

$$
y_{k}=\frac{1}{1+e^{\left(-z_{k}\right)}}
$$

2) The second type neuron

$\Sigma$ : (Linear function)

$$
z_{k}=w_{1} u_{1}+w_{2} u_{2}+w_{3} u_{3}+\cdots+w_{r} u_{r}-w_{0} \theta_{l}(r<p)
$$

$f:$ (Nonlinear function)

$$
y_{k}=\frac{1}{1+e^{\left(-z_{k}\right)}}
$$

(2) Radial basis function neural network

1) The first type neuron

$\Sigma:$ (Nonlinear function)

$$
z_{k}=w_{1} u_{i}+w_{2} u_{j}+w_{3} u_{i} u_{j}+w_{4} u_{i}^{2}+w_{5} u_{j}^{2}-w_{0} \theta_{l}
$$

$f:$ (Nonlinear function)

$$
y_{k}=e^{\left(-z_{k}^{2}\right)}
$$

2) The second type neuron

$\Sigma$ : (Linear function)

$$
z_{k}=w_{l} u_{1}+w_{2} u_{2}+w_{3} u_{3}+\cdots+w_{r} u_{r}-w_{0} \theta_{l} \quad(r<p)
$$

$f:$ (Nonlinear function)

$$
y_{k}=e^{\left(-z_{k}^{2}\right)}
$$

(3) Polynomial neural network

1) The first type neuron

$\Sigma:$ (Nonlinear function)

$$
z_{k}=w_{l} u_{i}+w_{2} u_{j}+w_{3} u_{i} u_{j}+w_{4} u_{i}^{2}+w_{5} u_{j}^{2}-w_{0} \theta_{l}
$$

$f:$ (Linear function)

$$
y_{k}=z_{k}
$$

\section{2) The second type neuron}

$\Sigma$ : (Linear function)

$$
z_{k}=w_{1} u_{1}+w_{2} u_{2}+w_{3} u_{3}+\cdots+w_{r} u_{r}-w_{0} \theta_{l} \quad(r<p)
$$

$f:$ (Linear function)

$$
y_{k}=z_{k}
$$

Here, $\theta_{l}=1$ and $w_{i}(i=0,1,2, \ldots, 5)$ and $w_{i}(i=0,1,2, \ldots, r)$ are weights between the input and hidden layer. Weights $w_{i}(i=0,1,2, \ldots)$ in each neural network architecture are estimated by the principal component-regression analysis.

\section{[Estimation procedure of weight $w_{i}$ ]}

First, values of $z_{k}^{* *}$ are calculated for each neural network architecture as follows.

a) Sigmoid function neural network

$$
z_{k}^{* *}=\log _{e}\left(\frac{\phi^{\prime}}{1-\phi^{\prime}}\right)
$$

b) RBF neural network

$$
z_{k}^{* *}=\sqrt{-\log _{e} \phi^{\prime}}
$$

c) Polynomial neural network

$$
z_{k}^{* *}=\phi
$$

where $\phi$ is an output variable and $\phi^{\prime}$ is the normalized output variable whose values are between 0 and 1 . 


\section{[Principal component-regression analysis]}

Multi-colinearity is generated in the function $\Sigma$ of the neurons. In this study, the function $\Sigma$ is calculated using the principal component-regression analysis.

In the case of Eq.(2), orthogonal vector $\underline{\boldsymbol{y}}$ is calculated .

$$
\underline{\boldsymbol{v}}=C \cdot \underline{\boldsymbol{u}}
$$

Here, $\underline{\boldsymbol{\nu}}=\left(v_{1}, v_{2}, \ldots, v_{5}\right), \underline{\boldsymbol{u}}=\left(u_{i} u_{j}, u_{i} u_{j}, u_{i}^{2}, u_{j}^{2}\right)$

$\underline{\boldsymbol{v}}$ is orthonormal vectors and $C$ is orthonormal matrix. $C$ is calculated using the following eigenvalue equation.

$$
R \cdot C=C \cdot \Lambda
$$

Here, $R$ is a correlation matrix. Then, variable $z_{k}$ is calculated using orthogonal regression analysis.

$$
\begin{aligned}
z_{k} & =\underline{\boldsymbol{w}}^{\mathbf{T}} \cdot \underline{\boldsymbol{y}} \\
& =w_{1} v_{1}+w_{2} v_{2}+\ldots+w_{5} v_{5}
\end{aligned}
$$

Using the principal component-regression analysis, variable $z_{k}$ in the function $\Sigma$ is calculated without multicolinearity. In (19), useful orthogonal variables $v_{i}(i=1,2, \ldots, 5)$ are selected by stepwise regression analysis ${ }^{9}$ using $\mathrm{AIC}^{7}$ or $\mathrm{PSS}^{8}$ criterion.

$L$ neurons having the smallest AIC or PSS values are selected for three types of neuron architectures. The output variables $y_{k}$ of $L$ selected neurons for three types of neuron architectures are set to the input variables of the neurons in the output layer.

\subsubsection{Output layer}

For three types of neural network, the outputs $y_{k}$ of the neurons in the hidden layer are combined by the following linear function.

$$
\phi^{*}=a_{0}+\sum_{k=1}^{L} a_{k} y_{k}
$$

Here, $L$ is the number of combinations of the input variables and $y_{k}$ is the intermediate variables. The optimal intermediate variables $y_{k}$ are selected by stepwise regression analysis ${ }^{9}$ using $\mathrm{AIC}^{7}$ or $\mathrm{PSS}^{8}$ criterion. Eq. (20) is calculated for three types of neural network architectures. The neural network architecture which has smallest AIC or PSS value is selected. Then, the estimated output values $\phi^{*}$ which is selected in the output layer is used as the feedback value.

\subsection{Second and subsequent loop calculations}

First, the estimated output value $\phi^{*}$ is combined with the input variables $x$ and all combinations between the estimated output value $\phi^{*}$ and the input variables $x$ are generated. The same calculation as the first feedback loop is iterated. When AIC or PSS value of the linear function in (20) is not decreased, the loop calculation is terminated and the complete neural network architecture is organized by the $L$ selected neurons in each feedback loop.

\section{Application to the medical image diagnosis of lung cancer}

In this study, the lung cancer regions were automatically recognized using the deep feedback GMDH-type neural network and these regions were extracted. Multi-detector row CT (MDCT) images of the lung were used in this study.

\subsection{Results of the medical image recognition by the deep feedback GMDH-type neural network}

The MDCT image shown in Fig.3 which was extracted from the MDCT image in Fig.2, was used for organizing the neural network. $x$ and $y$ coordinates and the statistics of the image densities in the neighboring regions of the $N \times N$ pixels at the positions of the learning points are used as the input variables of the neural network. Only five input variables which are the mean, the standard deviation, the variance and $x$ and $y$ coordinates were automatically selected as useful input variables. The output value of the neural network is zero or one. When $N \times N$ pixel region is contained in the lung cancer and blood vessel regions, the neural network set the pixel value at the center of the $N \times N$ pixel region to one and this pixel is shown as the white point. The neural networks were organized when the values of $N$ were from 3 to 10 . It was determined that when $N$ was equal to 3 , the neural network architecture had the smallest recognition error. Five useful neurons were selected in each hidden layer. Fig.4 shows the PSS values of the three types of neurons in the second layer. The RBF neural network architecture was selected by the deep GMDH-type neural network algorithm. Fig.5 shows the variation of PSS values in each layer. PSS values decreased gradually and very small PSS value was obtained at the seventh feedback loop calculation. The lung cancer and blood vessel regions were recognized by using the organized neural network and 
was extracted automatically. Fig.6 shows the output image of the deep feedback GMDH-type neural network. This output image was processed by the post-processing analysis. In the post-processing, the small isolated regions were eliminated and the outlines of the lung cancer regions were expanded outside by $N / 2$ pixels. Fig. 7 shows the output image after this processing. In order to check the matching between the original image and the output image of the neural network, the output image was overlapped on the original image in Fig.3. The overlapped image is shown in Fig.8. From Fig.8, we can see that the output image was very accurate. Fig.9 shows the extracted gray scale lung cancer image. These image processing were carried out for all slices by the organized deep feedback GMDH-type neural network and the 3-dimensional lung cancer image was generated as shown in Fig.10.

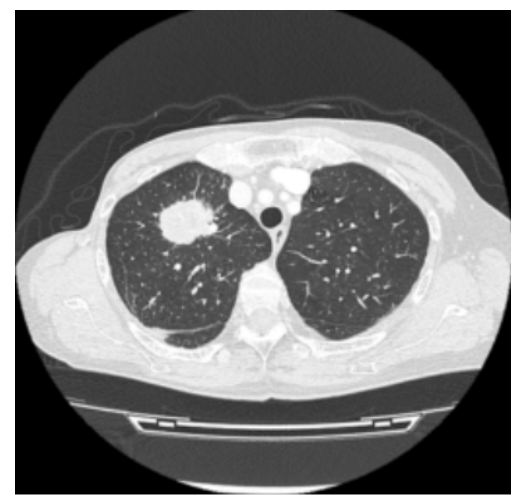

Fig.2 Original image

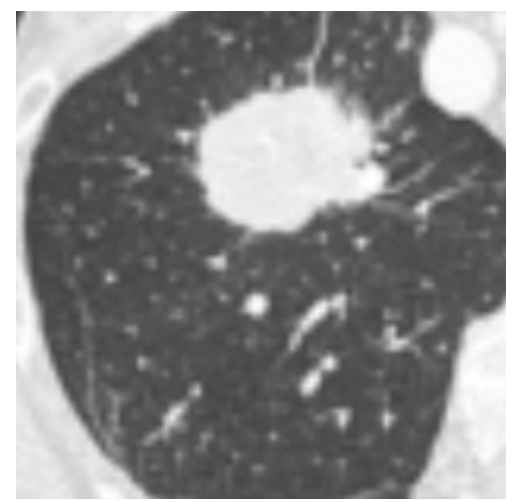

Fig.3 Original image extracted from Fig.2

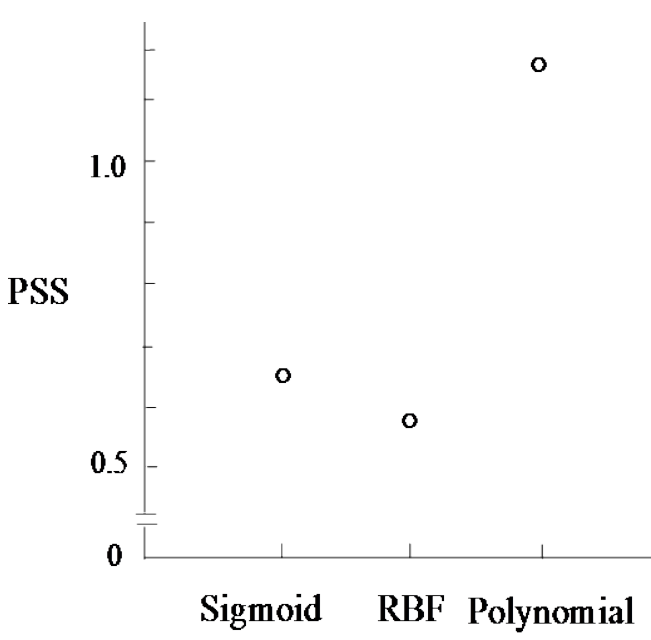

Fig. 4 PSS values of three types of neurons

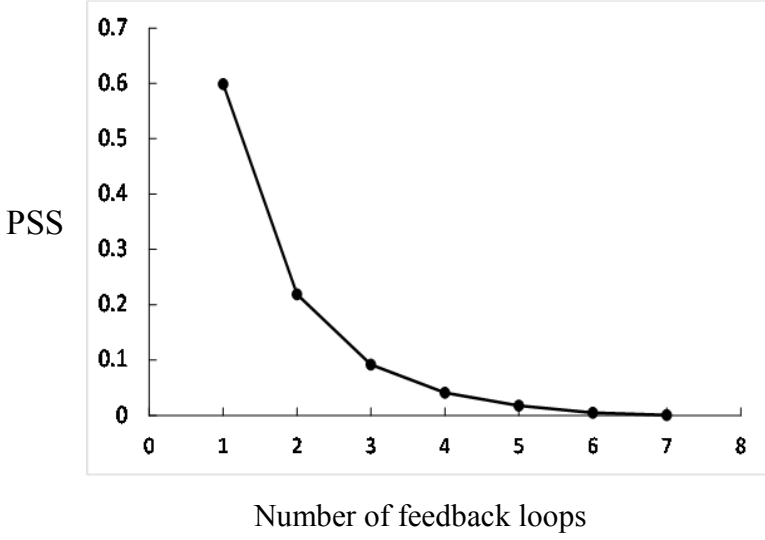

Fig. 5 Variation of PSS value in each feedback loop

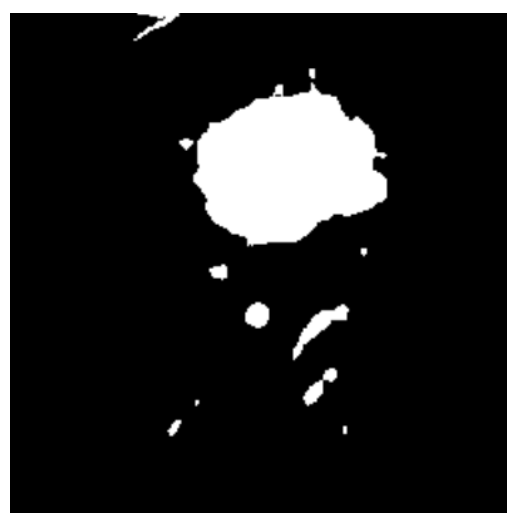

Fig. 6 Output image 


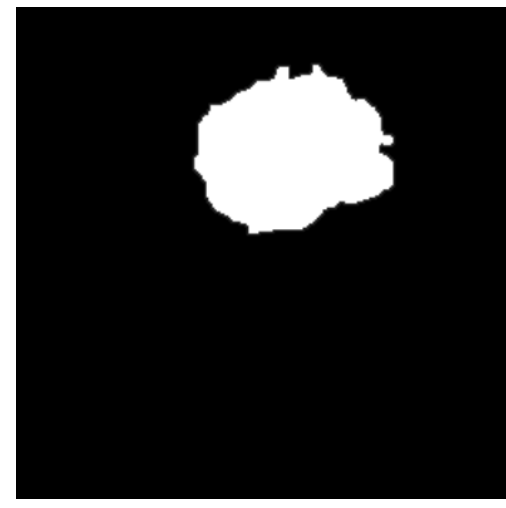

Fig.7 Output image after the post processing

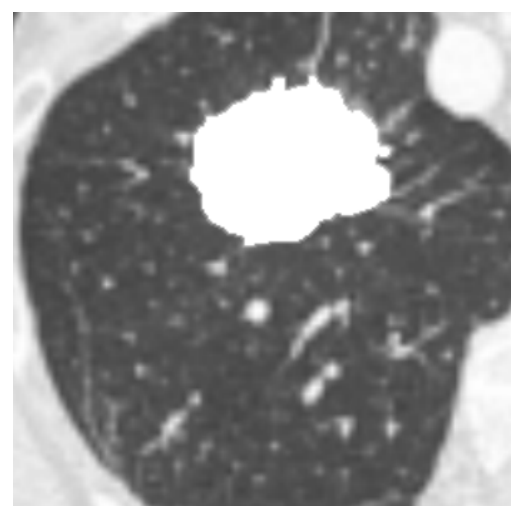

Fig. 8 Overlapped image

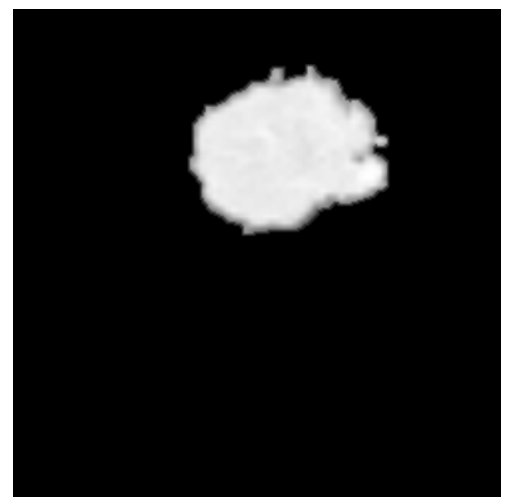

Fig. 9 Extracted gray scale image of lung cancer

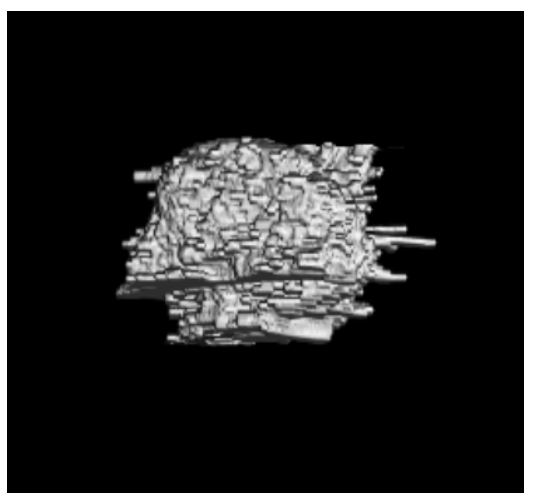

Fig.10 The 3-dimensional image of lung cancer

\subsection{Extraction by the conventional neural network using sigmoid function.}

A conventional neural network trained using the back propagation algorithm was applied to the same recognition problem. The learning calculations of the weights were iterated changing structural parameters such as the number of neurons in the hidden layer and the initial values of the weights. The output images, when the numbers of neurons in the hidden layer $(m)$ are 5, 7 and 9, are shown in Fig.11. These images contain more regions which are not part of the lung cancer and blood vessel regions, and the outlines of the lung cancer are not extracted with required clarity compared with the output images obtained using the deep feedback GMDH-type neural network algorithm, which are shown in Fig.6.

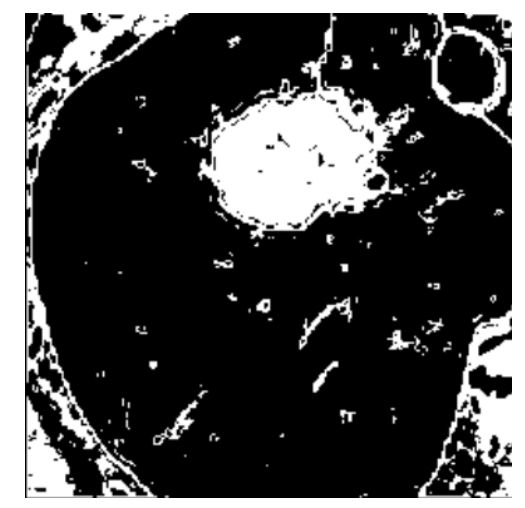

(a) $m=5$ 


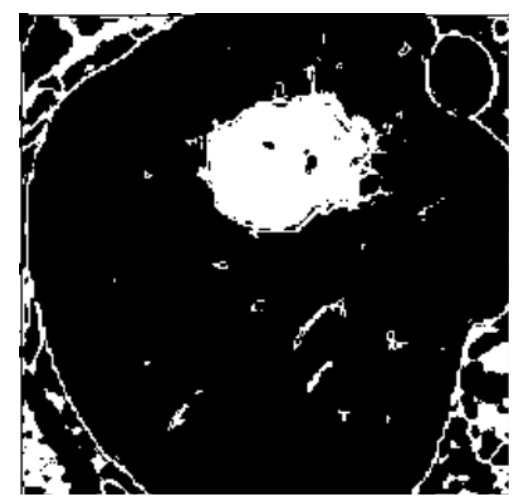

(b) $m=7$

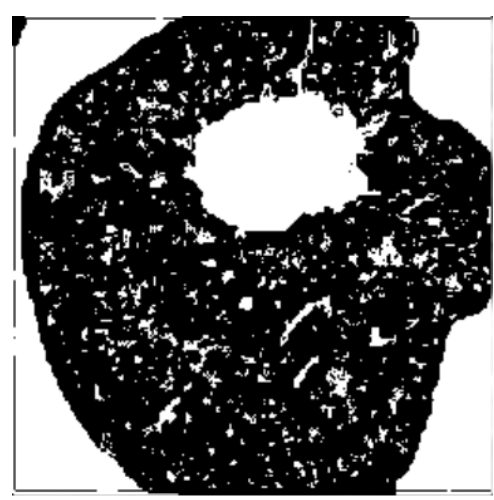

(c) $m=9$

Fig. 11 Output images of the conventional sigmoid function neural networks

\section{Conclusions}

In this paper, the deep feedback GMDH-type neural network algorithm was applied to the medical image recognition of lung cancer regions and the results were compared with those of the conventional sigmoid function neural network trained using the back propagation algorithm. It was shown that the deep feedback GMDH-type neural network algorithm was a useful method for the medical image diagnosis of the lung cancer because the deep feedback neural network architecture is automatically organized so as to minimize the prediction error criterion defined as AIC or PSS.

\section{Acknowledgment}

This work was supported by (JSPS) KAKENHI 26420421 .

\section{References}

1. T. Kondo, J. Ueno and S. Takao, Deep feedback GMDHtype neural network using principal componentregression analysis and its application to medical image recognition of abdominal multi-organs, Journal of Robotics, Networking and Artificial Life 2(2)(2015) 94 99.

2. T. kondo, J. Ueno and S. Takao, Medical image recognition of heart regions by deep multi-layered GMDH-type neural network using principal componentregression analysis, Journal of Robotics, Networking and Artificial Life, 2(3)(2015) 166-172.

3. T. Kondo, J. Ueno and S. Takao, Logistic GMDH-type neural network using principal component-regression analysis and its application to medical image diagnosis of lung cancer, Artificial Life and Robotics 20(2)(2015) 137144.

4. T. Kondo, J. Ueno and S. Takao, Medical image diagnosis of liver cancer by hybrid feedback GMDH-type neural network using principal component-regression analysis, Artificial Life and Robotics 20(2)(2015) 145151.

5. A. G. Ivakhnenko, Heuristic self-organization in problems of engineering cybernetics, Automatica 6(2)(1970) 207-219.

6. S. J. Farlow ed., Self-organizing methods in modeling, GMDH-type algorithm, New York: Marcel Dekker Inc., 1984.

7. H. Akaike, A new look at the statistical model identification, IEEE Trans. Automatic Control AC-19 (6) (1974) 716-723.

8. H. Tamura, T. Kondo, Heuristics free group method of data handling algorithm of generating optimum partial polynomials with application to air pollution prediction, Int. J. System Sci. 11 (9) (1980) 1095-1111.

9. N. R. Draper and H. Smith, Applied Regression Analysis, New York: John Wiley and Sons, 1982. 